\title{
Factibilidad de una comercializadora hortícola de economía solidaria en el Distrito de Riego del Alto Chicamocha
}

\section{Feasibility of a cooperative commercial association for horticultural products in the Distrito de Riego del Alto Chicamocha}

LUZ MARINA GUTIÉRREZ M. ${ }^{1,3}$

LUIS FELIPE RODRÍGUEZ C. ${ }^{1}$

LILIA TERESA BERMÚDEZ C. ${ }^{2}$

Comercializadora de frutas $y$ hortalizas en la Central Minorista del municipio de Duitama, Boyacá. Foto: R. Pedraza

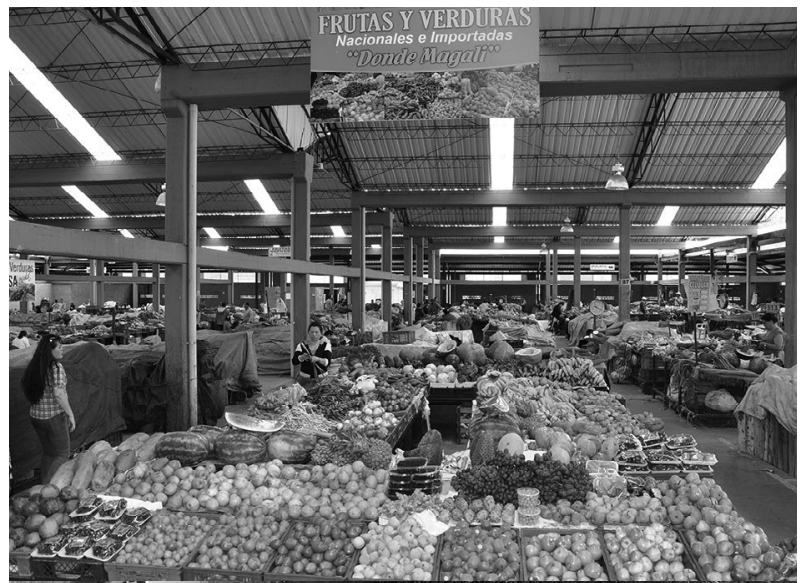

\section{RESUMEN}

Como un aporte a la solución de uno de los problemas de los productores hortícolas del Distrito de Riego del Alto Chicamocha (Duitama, Colombia), consistente en la carencia de una organización empresarial que comercialice los productos en mejores condiciones de mercado, se realizó una encuesta entre productores, comercializadores, consumidores y se analizaron todos los factores que tienen marcada influencia en el proceso de producción y comercialización. De acuerdo con el resultado, se hace necesario la organización de una empresa de tipo solidario cuya ubicación sea la ciudad de Duitama, donde los productores se asocien desde la producción hasta la comercialización para lograr la regulación de precios, la disminución de intermediarios y la obtención de mejores ingresos, al tiempo que desarrollen procesos productivos de acuerdo con las necesidades del consumidor, adelantando labores de poscosecha como adecuación y empaque. La empresa propuesta será de servicios y en consecuencia realizará una función de agente comercializador entre el asociado y el consumidor final.

\footnotetext{
Palabras clave adicionales: horticultores, mercadeo, organización, empresa asociativa, cadena productiva.

Facultad de Agronomía, Departamento de Agronomía, Universidad Nacional de Colombia, Bogotá (Colombia).

2 Facultad Seccional Duitama, Escuela de Administración Industrial, Universidad Pedagógica y Tecnológica de Colombia (UPTC), Duitama (Colombia).

3 Autor para correspondencia.luzmagum@gmail.com
} 


\section{ABSTRACT}

In response to the need for a commercial organization that would facilitate the marketing of horticultural products in the Distrito de Riego del Alto Chicamocha (Irrigation District of the Upper Chicamocha River) in Boyacá (Colombia), a survey was conducted among producers, dealers and consumers, which analyzed all the factors that have noticeable influence on the production and commercialization process. According to the results, it is necessary to establish a cooperative-type service organization, which would act as the marketing agent between the associates and the consumers. This organization should be located in the municipality of Duitama for strategic marketing and, therefore, the ability to contribute to the associability of the producers from production to marketing. Thereby, the producers will be able to focus their efforts to meet the needs of the market, both in quality and quantity; regulating prices, decreasing intermediaries and increasing income.

Additional key words: horticulturists, marketing, organizations, commercial association, productive chain.

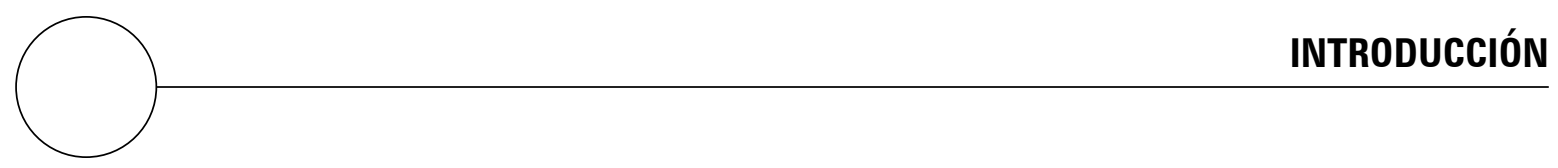

El presente trabajo de investigación se realizó como un aporte a la solución de uno de los problemas más sentidos por los productores hortícolas del Distrito de Riego del Alto Chicamocha (DRACH), consistente en la carencia de una organización empresarial que les permita comercializar sus productos adecuadamente y mejorar condiciones de mercado.

Los productores hortícolas del DRACH poseen un sistema de producción estacional como consecuencia de la ausencia de una estrategia de planeación y programación de la producción y la ampliación del área y sus criterios racionales de uso de recursos, en algunos sistemas productivos como el de la cebolla de bulbo. En consecuencia, no existe una oferta permanente de productos, afectando negativamentela posibilidad de obtener mejores precios y efectuar un mercadeo eficiente.

Pese a que la zona cuenta con el recurso estratégico del riego y con las ventajas comparativas adicionales, como tierra plana y fértil, trayectoria en la actividad y ubicación favorable en rela- ción con los principales mercados, no se ha logrado construir una organización empresarial para la comercialización de los productos.

Actualmente, la comercialización de los productos del distrito se realiza en mercados locales (autoservicios y plazas de mercado) y en la Central de Abastos de Corabastos en Bogotá, así como en algunos mercados especializados, almacenes de cadena en los que se comercializan productos como la cebolla, la arveja, el brócoli y la lechuga y para los cuales en el canal de comercialización predominante sobresalen acopiadores, e intermediarios que colocan las condiciones de mercadeo en detrimento de los productores.

También es destacable la falta de experiencia y de organización de los productores, debido en parte al sistema de producción individual. No obstante en épocas anteriores se han dado algunos procesos de organización asociativa entre los usuarios para la comercialización, los cuales han fracasado por falta de pertenencia, liderazgo y baja capacidad empresarial. 
Por lo anterior se puede deducir que la creación de una empresa solidaria podría convertirse en una solución al problema del mercadeo, siempre y cuando se dé una apropiación del modelo de organización solidaria donde se trabaje asociativamente, tanto en la producción como en la comercialización, con lo que se logre la organización y planificación de la producción, se haga uso eficiente de los recursos agua y tierra, se reduzcan costos, se obtengan mejores precios y se acceda a mercados especializados, lo que permitiría también darle un valor agregado a los productos a través de la cadena productiva agroalimentaria.

El concepto de forma asociativa en el campo, según Murcia (1995), contempla todas aquellas unidades en las que se efectúan actividades de carácter colectivo en el proceso de alcanzar un objetivo. Este término comprende, por tanto, todos los tipos de cooperativas o formas comunitarias que tienen relación, directa o indirecta, con el sector primario de la economía y que se podrían localizar tanto en el campo de la producción como en el de los servicios de crédito, ahorro, consumo, comercialización etc.

De acuerdo con lo expresado por Pineda (2008), el logro de los objetivos institucionales en las empresas solidarias exige la implantación de la estructura organizacional que responda a la disponibilidad de recursos humanos y materiales y a las expectativas y aspiraciones del conjunto de asociados dentro de las posibilidades que brinde el entorno en el cual se desenvuelven.

Dentro de las fases para la creación de una empresa de economía solidaria se encuentra el estudio de factibilidad. Según ILPES (1973), se trata de ordenar las alternativas de solución para el proyecto, según ciertos criterios, tanto desde el punto de vista del empresario público o privado, como desde la perspectiva de la economía en su conjunto.

Como lo menciona Rojas (2007), los principales aspectos bajo los cuales se estudia un proyecto se refieren a los problemas técnicos, económicos, financieros, administrativos e institucionales, que pueden plantearse con distinto acento en cada estudio parcial que lo componen.

De acuerdo con ILPES (1973), la finalidad del estudio de mercados es probar que existe un número suficiente de individuos, empresas u otras entidades económicas que, dadas ciertas condiciones, presentan una demanda que justifica la puesta en marcha de un determinado programa de producción -de bienes o servicios- en un cierto periodo.

La descripción de la unidad productiva comprende dos conjuntos de elementos: un grupo básico que reúne los resultados relativos al tamaño del proyecto, su proceso de producción y su localización; y otro grupo de elementos complementarios que describe las obras físicas necesarias, la organización para la producción y el calendario de realización del proyecto. Esos dos conjuntos son interdependientes y se relacionan estrechamente con los estudios financiero y económico del proyecto y con los resultados alcanzados en el estudio de mercado (Córdoba, 2006).

Se incluye además en el estudio técnico el análisis de los costos del proyecto que resultan de las soluciones dadas a los problemas técnicos y económicos presentados en el mismo capítulo (DNP, 2012).

Prieto (2009) argumenta que el estudio legal busca determinar la viabilidad de un proyecto a la luz de las normas que lo rigen en cuanto a localización, utilización de productos, subproductos y patentes. También toma en cuenta la legislación laboral y su impacto a nivel de sistemas de contratación, prestaciones sociales y demás obligaciones laborales.

El estudio financiero, según lo planteado por Miranda (2011), comprende la inversión, la proyección de los ingresos y de los gastos y las formas de financiamiento que se prevén para todo el 
periodo de su ejecución y de su operación. Debe mostrar en forma ordenada las fuentes de los recursos financieros que se utilizarán y su distribución en los diversos usos que comprende el proyecto o en otras palabras, el origen y el destino de los recursos. El estudio deberá demostrar que el proyecto puede realizarse con los recursos financieros disponibles. Así mismo se deberá evaluar la decisión de comprometer dichos recursos en el proyecto en comparación con otras posibilidades conocidas de colocación (Núñez, 2007)

De acuerdo con Murcia et al. (2009), el estudio ambiental se centra principalmente en dos temas: el análisis del impacto del proyecto sobre el medio ambiente (con el fin de minimizar deterioros causados por el proyecto) y el análisis del efecto del entorno sobre el proyecto (para aportar a la adecuada formulación del mismo).

Un aspecto complementario fundamental es la evaluación financiera que, según lo planteado por Mokate (2009), analiza el proyecto a la luz de su retorno financiero. Así, se enfoca en el análisis del grado en que el proyecto cumple el objetivo de generar un retorno a los diferentes actores que participan en su ejecución o financiamiento (Naranjo, 2008).

La evaluación económica trabaja los flujos de ingresos y egresos con los precios vigentes en los mercados, y según el objetivo del análisis, puede ajustarse para eliminar los efectos de la inflación sobre dichos precios. Típicamente toma como criterio de selección el valor presente neto (VPN) o la tasa interna de retorno (TIR) (Ramírez, 2011).

La evaluación económica, según lo expresa el DNP (2012), compara los costos y los beneficios del proyecto desde el punto de vista de la economía en su conjunto. El objetivo de este tipo de evaluación se centra en la medición de la contribución del proyecto al bienestar económico del país.
La evaluación social está muy ligada con la evaluación económica y de acuerdo con lo planteado por Mokate (2009), también examina el proyecto desde el punto de vista nacional, evaluando no solo su contribución al bienestar económico, sino también los objetivos de la política social de redistribución de ingresos y riquezas, por lo cual, entonces, la evaluación social es una extensión de la evaluación económica.

El objetivo general de este estudio de caso se centra en realizar la formulación y evaluación de la factibilidad económico-administrativa, de una organización empresarial de carácter solidaria como alternativa, para la comercialización eficiente de los productos hortícolas del Distrito de Riego del Alto Chicamocha, teniendo en cuenta las ventajas económicas, organizativas y de gestión que este modelo ofrece.

\section{METODOLOGÍA Y PROCEDIMIENTOS DE INVESTIGACIÓN}

Para el desarrollo de este estudio de caso se utilizó un diseño metodológico no experimental de carácter descriptivo.

El proyecto de investigación se adelantó en la zona del Distrito de Riego del Alto Chicamocha, ubicado en la cuenca alta del río Chicamocha, en jurisdicción de 11 municipios, dentro de los que se desatacan: Paipa, Duitama, Santa Rosa de Viterbo, Tibasosa, Nobsa, Firavitoba y Sogamoso.

Las fuentes de información primaria fueron los productores hortícolas a quienes se les aplicó en el año 2011 un formulario previamente elaborado y probado. De igual manera se realizó una encuesta a los consumidores en los autoservicios de la ciudad de Duitama y algunos de Sogamoso.

Las fuentes de información secundarias las constituyeron fundamentalmente los informes institucionales (FAO, Usochicamocha, Fondo de Fomento Hortofrutícola, documentos SENA, 
trabajos de grado de la UPTC y el CCI [2000]). El formulario, que se aplicó a los productores hortícolas en el Distrito de Riego, incluyó varios aspectos: caracterización física de la agroempresa de producción hortícola, aspectos socioeconómicos del productor, aspectos de comercialización $\mathrm{y}$ organizacionales, y expectativas de vinculación a la organización.

Las notas de entrevistas, se utilizaron para consignar la información aportada en las entrevistas realizadas a un total de 10 funcionarios de entidades como: Usochicamocha, CCI y gerentes de organizaciones solidarias agropecuarias, directivos del Plan Alimentario para Aprender (PAPA).

Las poblaciones objetivo estuvieron constituidas así:

- Población de productores. Esta población objeto del estudio está constituida por los productores de hortalizas usuarios del DRACH, ubicados en los 2.684 predios con riego instalado (tabla 1).

- Población de consumidores. Constituida por los consumidores de los productos selecciona- dos en los principales autoservicios de la ciudad de Duitama como El Paraíso, Éxito, Mercópolis, Pasadena, y en Sogamoso, La Canasta y Olímpica, de las cuales se tomó una muestra de 10 personas por supermercado.

Para adelantar la toma de información, se procedió a la selección de una muestra constituida por el 5\% (131) de productores, a través del muestreo aleatorio estratificado con afijación proporcional, teniendo en consideración el número de predios de cada una de las unidades de riego.

También se seleccionó una muestra representativa de 10 consumidores asiduos clientes por cada almacén de cadena en las ciudades de Duitama y Sogamoso. De igual manera se investigó al consumidor institucional representado por el plan departamental para la alimentación, plan PAPA, por la magnitud de demanda por los productos hortícolas, información que se codificó y tabuló de conformidad con las temáticas básicas de estadística descriptiva.

Es de destacar que se utilizó la metodología de investigación acción participativa para acceder a la información primaria con los productores.

Tabla 1. Población de predios con riego del distrito y composición de la muestra investigada, según Usochicamocha (2011).

\begin{tabular}{|l|c|c|c|}
\hline \multicolumn{1}{|c|}{ Unidades de riego } & Número de predios & Porcentaje & $\begin{array}{c}\text { Muestra } \\
\text { (5\% de los predios) }\end{array}$ \\
\hline Ayalas & 202 & 7,5 & 10 \\
\hline Cuche & 272 & 10,1 & 14 \\
\hline Duitama & 207 & 7,7 & 8 \\
\hline Las Vueltas & 158 & 5,9 & 19 \\
\hline Ministerio & 371 & 13,8 & 20 \\
\hline Moniquirá & 390 & 14,5 & 10 \\
\hline Pantano de Vargas & 194 & 7,2 & 30 \\
\hline San Rafael & 681 & 25,4 & 5 \\
\hline Surba & 209 & 7,8 & 4 \\
\hline Tibasosa & 363 & 13,5 & 131 \\
\hline Total & 2.684 & 100 & \\
\hline
\end{tabular}


Se realizaron 10 encuestas a los encargados de la sección de fruver de los autoservicios que presen$\tan$ las mayores ventas ubicados en Sogamoso y Duitama, con el fin de tener un estimativo de la posible demanda de los productos de la empresa. Así mismo se entrevistaron los encargados de la comercialización del plan PAPA.

Para la evaluación financiera del proyecto se recurrió al uso de indicadores financieros como la tasa interna de retorno (TIR), relación beneficio/ costo $(\mathrm{B} / \mathrm{C})$, y valor presente neto (VPN), además se llevó a cabo el respectivo análisis de sensibilidad.

$\operatorname{TIR}=\frac{\Sigma \mathrm{BN}_{\mathrm{t}}}{\left(1+\mathrm{i}_{\mathrm{op}}\right)} \mathrm{t}=0$

Relación $\mathrm{B} / \mathrm{C}=\mathrm{VPB} / \mathrm{VPC}$

Donde: $\mathrm{VPB}=$ valor presente de los beneficios brutos

$\mathrm{VPC}=$ valor presente de los costo

$\mathrm{VPN}=\frac{\left(\Sigma\left(\mathrm{BN}_{\mathrm{t}}\right)\right.}{\left(1+\mathrm{i}_{\mathrm{op}}\right) \mathrm{t}}$

Donde: $\mathrm{BN}_{\mathrm{t}}=$ beneficio neto en el periodo $\mathrm{t}$

$\mathrm{i}_{\text {op }}=$ tasa de interés de oportunidad

$\mathrm{T}=$ ultimo periodo de la vida del proyecto

$\mathrm{t}=1,2,3, \ldots \mathrm{T}$

\section{ANÁLISIS Y DISCUSIÓN DE RESULTADOS}

Teniendo en cuenta el resultado de la encuesta aplicada a los productores, el tipo de agro empresa que predomina en sus explotaciones es la familiar (49,2\%), individual $(22,6 \%)$ y se destaca la sociedad o compañía, con el 33\%, forma que se caracteriza porque uno de los socios coloca la tierra y el otro los insumos, modelo que predomina en las explotaciones de cebolla.

Actualmente, los propietarios de las fincas no arriendan sin conocer cómo se utilizará la tierra, por tanto, en los contratos se especifican los cultivos que se van a desarrollar, dado el hecho de que los contratos son por varios años.

Las actividades principales en el DRACH son: agricultura, ganadería, comercio y transporte. En relación con la generación de ingresos, el 74,2\% de los productores tiene como actividad exclusiva la agricultura, unida esta con la ganadería en un $24,19 \%$, También se encuentran algunos productores que laboran en la agricultura asociada con el comercio y otras actividades diferentes (Usochicamocha, 2011).

El DRACH cuenta con una población con vocación agropecuaria y mano de obra suficiente y calificada para el desarrollo de las actividades productivas del agro. Según lo expresado por los productores, alrededor de las explotaciones agrícolas se mueven actividades comerciales como la venta de muebles y enseres, venta de predios, agro tiendas, supermercados, venta de insumos etc.

El área de influencia del distrito cuenta con servicios de educación, de buena calidad en todos los niveles: básica, media vocacional, bachillerato y universitaria, lo mismo que se destaca la presencia de entidades de capacitación técnica como el SENA.

Según documento informativo del DRACH (Usochicamocha, 2011), la actividad agrícola del distrito cubre aproximadamente unas 1.500 ha $(37,5 \%)$ del total de hectáreas con riego en donde se producen $240.000 \mathrm{t}$ de alimentos/año, destacándose la producción de cultivos como cebolla de bulbo, arveja, remolacha, repollo, espinaca, zanahoria, brócoli, frutales, fríjol, papa y acelga. 
En los últimos años se observa que más tierras se han dedicado a la agricultura en especial por el auge de cultivos como la cebolla de bulbo y la implementación de otros como el calabacín y la habichuela.

Según las estadísticas de Agronet (2013), los rendimientos por hectárea de brócoli en Boyacá en el año 2010 fueron de 16,2 t, mientras que los rendimientos reportados por el DRACH se encuentran en $22 \mathrm{t}$ con unos costos de producción de $\$ 7.650 .000 /$ ha y la participación del cultivo en la producción nacional de 1,98\%. Estas diferencias en rendimiento por hectárea se deben a las excelentes condiciones de producción de la zona del distrito para este tipo de hortaliza.

De igual manera, los rendimientos por hectárea de cebolla de bulbo en Boyacá en el año 2010 fueron de 24,3 t mientras que el rendimiento por hectárea es de $30 \mathrm{t}$, (tabla 2) en el DRACH en condiciones óptimas de producción y la participación en la producción nacional fue del 50,7\%, debido a que las áreas de producción disponen de una buena dotación de recursos como la tierra y el riego, lo que les permite mejorar su productividad.

Según el resultado de la encuesta a los productores, el $70 \%$ de estos son propietarios de la tierra y la cultivan. El 18,8\% es arrendatario y el 11,2\% tiene como modalidad de tenencia la denominada compañía o sociedad caracterizada por que uno de los socios es dueño de la tierra. En el dis- trito de riego predominan las explotaciones con características de minifundio, ya que el $91 \%$ poseen un tamaño de menos de 5 ha, entre 5 y 10 ha el $6 \%$ y de más de 10 ha el 3\% (tabla 3).

El sistema de producción en el distrito de riego es el de monocultivo, predominando el cultivo de la cebolla de bulbo, rotado con otras hortalizas, en especial, repollo, brócoli, arveja y espinaca. Se puede observar que cada vez más el suelo se dedica al cultivo de la cebolla, producto que deteriora la capacidad del mismo, a pesar de que se realiza en un sistema de rotación con otras hortalizas. Ya se observan áreas de cultivo abandonadas por la presencia de patógenos resistentes a los agroquímicos.

Los productores del DRACH comercializan sus productos básicamente a través de intermediarios a quienes venden a crédito o en plazas de mercado, donde por lo general lo venden de contado.

De acuerdo con lo expresado por los productores, el $79,4 \%$ cuenta con recursos propios para desarrollar la producción y el 20,6\% se financian con crédito. Algunos alternan las dos formas de financiación.

Es de destacar que los principales problemas del mercadeo de los productos hortícolas en el distrito están relacionados con los bajos precios, la posición dominante de los intermediarios, la ausencia de canales de comercialización estructurados

Tabla 2. Productividad parcial de la tierra y costos de producción por ha y año de los principales cultivos del DRACH, 2011.

\begin{tabular}{|l|c|c|}
\multicolumn{1}{|c|}{ Sistema de producción } & $\begin{array}{c}\text { Productividad de la tierra } \\
\left(\mathrm{t} \mathrm{ha}^{-1}\right)\end{array}$ & $\begin{array}{c}\text { Costos de producción } \\
\text { (\$/ha) }\end{array}$ \\
\hline Cebolla cabezona & 30 & 13.000 .000 \\
\hline Lechuga & 12 & 8.909 .000 \\
\hline Coliflor & 30 & 9.700 .000 \\
\hline Brócoli & 22 & 7.650 .000 \\
\hline Arveja & 4,5 & 5.000 .000 \\
\hline Repollo & 25 & 3.400 .000 \\
\hline
\end{tabular}


Tabla 3. Clasificación por tamaño de las explotaciones del DRACH, 2011.

\begin{tabular}{|c|c|}
\hline Tamaño & Porcentaje \\
\hline$<1$ ha & 46 \\
\hline 1 - 5 ha & 45 \\
\hline 5 - 10 ha & 6 \\
\hline$>10$ ha & 3 \\
\hline
\end{tabular}

y la falta de una organización para la comercialización por lo que los productores se encuentran a merced de los intermediarios, quienes colocan el precio del producto.

En aras de mejorar las condiciones económicas del producto y su comercialización, y a partir de los resultados de la encuesta aplicada, el 90\% de los productores están dispuestos a adaptarse a un sistema unificado de cultivos que les permita tener mayores volúmenes y calidades de este.

Los productos se venderán al programa plan PAPA a través del cual se cubre a 23 municipios en Boyacá y que está dirigido a suministrar alimentación a los niños de las escuelas y al adulto mayor. De lo anterior, se espera que el servicio que la empresa ofrecerá a sus asociados, es el de constituirse en un agente intermediario entre el asociado y el consumidor final, en este caso el plan alimentario para Boyacá para lo cual la empresa recibirá los productos en consignación y cobrará a los asociados una tarifa por el servicio (tabla 4).

Para el desarrollo del proyecto de la empresa co-

\section{Tabla 4. Tipo de servicio que prestará la empresa de comercialización a los productores del DRACH, 2011.}

\begin{tabular}{|l|c|c|}
\hline \multirow{2}{*}{\multicolumn{1}{|c|}{ Servicio }} & \multicolumn{2}{c|}{ Productores } \\
\cline { 2 - 3 } Compra-venta & 37 & 28,2 \\
\hline $\begin{array}{l}\text { Adecuación del } \\
\text { producto }\end{array}$ & 10 & 7,6 \\
\hline Comisionista & 78 & 59,5 \\
\hline Otro & 6 & 4,6 \\
\hline Total & 131 & 100,0 \\
\hline
\end{tabular}

mercializadora de productos hortícolas, se ha tenido en cuenta el hecho de que los asociados suministrarán, según proyección de la producción, alrededor de $141.120 \mathrm{~kg}$ en el primer año entre brócoli y cebolla, es decir unos $11.760 \mathrm{~kg}$ mensuales. De acuerdo con la demanda del mercado se fija un incremento del 15\% aproximadamente a partir del segundo año en adelante, es decir $162.288 \mathrm{~kg}$ anuales para el tercero, $186.631 \mathrm{~kg}$ para el cuarto y para el quinto año $214.625 \mathrm{~kg}$.

De acuerdo con los resultados de la encuesta, el $90 \%$ de los productores del DRACH comercializan sus productos básicamente a través de intermediarios, quienes negocian en el predio el producto abonando un porcentaje del valor de la compra y cancelando el resto generalmente a 30 días. Otra forma de comercialización es en la plaza de mercado donde por lo general venden de contado. Los productores en su mayoría venden en el momento de la cosecha y tan solo un 10\% semanalmente, debido a que no manejan siembras escalonadas.

Según el estudio de mercado, de la producción del DRACH, el 36\% se comercializa en mercados de Bogotá, desde donde se distribuye hacia la Costa Atlántica, Bolívar, Magdalena y Sucre; el $5 \%$ va directamente hacia Santander y un 59\% se queda para consumo en el departamento.

E1 $80 \%$ de los productores hortícolas están interesados en conformar la empresa comercializadora de productos hortícolas en el distrito, de los cuales $60 \%$ se inclina por una cooperativa integral de comercialización y $20 \%$ una organización asociativa de productores con el fin de mejorar las condiciones de mercadeo de sus productos.

De acuerdo con la encuesta realizada en los autoservicios y almacenes de cadena, de las ciudades de Duitama y Sogamoso, se afirma que sus proveedores por lo general son los intermediarios, acopiadores y productores que garantizan el abastecimiento en periodos de baja disponibili- 
dad, con el fin de mantener en los puntos de venta la mayor regularidad posible, de su portafolio de productos ofertado a sus clientes.

Con respecto a la configuración del canal de comercialización mediante el cual se efectuará el traslado del producto hasta el consumidor: el primer eslabón será el productor quien entrega a la comercializadora en consignación la producción de hortalizas. La empresa comercializadora a su vez, como agente comercializador, entrega el producto al consumidor institucional, en este caso al plan de alimentación PAPA, quien a su vez entrega a los comedores escolares de los distintos municipios establecidos en dicho programa. A partir de los resultados de la encuesta, el $48 \%$ de los productores se encuentran interesados en que el proyecto se ubique en la ciudad de Duitama.

Según Córdoba (2006), está definido el hecho de que el tamaño del proyecto está relacionado con el tamaño del mercado, en este caso la demanda actual por las hortalizas; de esto se deduce que la comercializadora tendrá una capacidad para recepcionar alrededor de 11,2 t mes $^{-1}$ entre cebolla cabezona $\left(7.200 \mathrm{~kg} \mathrm{mes}^{-1}\right)$ y brócoli $\left(4.000 \mathrm{~kg} \mathrm{mes}^{-1}\right)$, de acuerdo con la demanda del programa de alimentación del departamento de Boyacá, plan PAPA. La capacidad máxima de recepción de hortalizas se presenta entre febrero y noviembre, meses de labores académicas.

Para el establecimiento de la comercializadora se requiere disponer de las siguientes instalaciones: una bodega adecuada para el área administrativa y de servicios de recepción del producto, clasificación, zona de empaque y almacenamiento. Esta bodega se encuentra disponible en Duitama en el área urbana con un área inicial de $126 \mathrm{~m}^{2}$, dentro de la cual se adecuará un mezanine (división de un local o bodega en donde generalmente se ubican las oficinas) de $90 \mathrm{~m}^{2}$ para administración y donde también se puede almacenar los empaques y las canastillas.
Teniendo en cuenta el proceso de comercialización de las hortalizas, el cual lleva implícitas las etapas desde la recepción de las mismas hasta la entrega al consumidor final, en la tabla 5 se describen los costos asociados a cada una de ellas.

De acuerdo con los resultados de la encuesta, el $59,5 \%$ de los productores se muestra interesado en que se constituya una cooperativa integral de comercialización de productos hortícolas, de la misma manera un 19,1\% sugiere que sea una organización asociativa de productores, en tanto que el $4,6 \%$ propone una empresa asociativa de trabajo y el $11,4 \%$ manifiesta no inclinarse por ningún tipo de organización. La decisión final se materializó en establecer una cooperativa integral, en razón a que se considera que puede realizar dos o más actividades conexas y complementarias entre sí, producción, distribución, consumo y prestación de servicios y tendrá por razón social "Coophortiboy", con sede en la ciudad de Duitama. El 80,6\% de los productores de hortalizas del distrito de riego, manifiesta que estarían dispuestos a participar de este tipo de organización asociativa para la comercialización de sus productos.

Coophortiboy será una cooperativa integral de comercialización, organización del sector solidario regida por la Ley 79 del 88, cuya creación se efectuará ante la Cámara de Comercio de Duitama, los dignatarios elegidos en la asamblea de constitución y los estatutos de la misma.

La información financiera básica para adelantar la respectiva evaluación de este proyecto se presenta en la tabla 6.

La evaluación financiera y el análisis de rentabilidad del proyecto se fundamentan en el flujo de fondos (tabla 6). De acuerdo con la coyuntura económica del país, en este proyecto se utilizó una tasa de oportunidad del mercado del 10\%, la cual nos arrojó una VAN de $\$ 9.520 .835$, lo que 
Tabla 5. Costos del servicio de comercialización de "Coophortiboy", 2011.

\begin{tabular}{|c|c|c|c|c|c|}
\hline \multirow{2}{*}{$\begin{array}{c}\text { Costos (\$) } \\
\text { Costos directos }\end{array}$} & \multicolumn{5}{|c|}{ Años } \\
\hline & 1 & 2 & 3 & 4 & 5 \\
\hline $\begin{array}{l}\text { Mano de obra } \\
\text { bodeguero }\end{array}$ & 5.592 .000 & 5.592 .000 & 5.592 .000 & 5.592 .000 & 5.592 .000 \\
\hline Acondicionamiento & 2.688 .000 & 3.252 .480 & 4.105 .882 & 4.721 .750 & 4.721 .750 \\
\hline Empaque & 1.140 .000 & 1.140 .000 & 1.140 .000 & 1.140 .000 & 1.140 .000 \\
\hline Transporte & 16.128 .000 & 18.480 .000 & 23.328.875 & 26.828 .125 & 26.828 .125 \\
\hline $\begin{array}{l}\text { Total costos } \\
\text { directos }\end{array}$ & 25.548 .000 & 28.464 .480 & 34.166 .757 & 38.281 .875 & 38.281 .875 \\
\hline \multicolumn{6}{|l|}{ Costos indirectos } \\
\hline Asistencia técnica & 7.200 .000 & 7.200 .000 & 7.200 .000 & 7.200 .000 & 7.200 .000 \\
\hline $\begin{array}{l}\text { Admon. } \\
\text { comercializadora }\end{array}$ & 26.952 .000 & 26.952 .000 & 26.952 .000 & 26.952 .000 & 26.952 .000 \\
\hline Depreciaciones & 1.121 .000 & 1.121 .000 & 1.121 .000 & 1.121 .000 & 1.121 .000 \\
\hline $\begin{array}{l}\text { Total costos } \\
\text { indirectos }\end{array}$ & 35.273 .000 & 35.273 .000 & 35.273 .000 & 35.273 .000 & 35.273 .000 \\
\hline Total costos & 60.821 .000 & 63.737 .480 & 69.439 .757 & 73.554 .875 & 73.554 .875 \\
\hline $\begin{array}{l}\text { Unidades } \\
\text { comercializadas }\end{array}$ & 134.400 & 147.840 & 186.631 & 214.625 & 214.625 \\
\hline Costo unitario & 452,54 & 431,12 & 372,07 & 342,71 & 342,71 \\
\hline
\end{tabular}

nos permite afirmar que el proyecto genera una rentabilidad favorable para su implementación.

Por su parte, la TIR muestra la rentabilidad propia de la inversión, lo que permite mantener la sostenibilidad del proyecto (Miranda, 2011). Es así que con una tasa de interés de oportunidad del $10 \%$ se alcanza una TIR del $21 \%$, es decir se encuentra por encima de la tasa de interés a la cual el inversionista puede conseguir los recursos financieros (tasa de interés de oportunidad) por lo tanto el proyecto resulta factible (tabla 7). Igualmente se realizó el análisis de sensibilidad, en el cual se plantean y comparan escenarios posibles para el proyecto, ante el comportamiento de algunas variables que puedan presentar modificaciones durante la vida del proyecto y que afectan directamente la rentabilidad del mismo (DNP, 2012).

Una vez realizado el análisis de sensibilidad (tabla 7 ), se puede concluir que el escenario dos es el que representa las mejores condiciones de rentabilidad, ya que se observa un leve incremento de los indicadores en relación con el escenario natural, así: una TIR del $32 \%$, frente al $21 \%$ se encuentra por encima de la tasa de oportunidad del mercado de $10 \%$, la VAN de 10.841.118 muestra la ganancia neta generada por el proyecto lo mismo que la relación $\mathrm{B} / \mathrm{C}$ de 1,069 y en consecuencia se puede afirmar que tanto el escenario inicial como el 2 ofrecen las mejores condiciones de adelantar el proyecto, no así el 1, donde la TIR no presenta su valor por ser muy pequeño y todos los indicadores de rentabilidad caen.

El estudio de factibilidad para el montaje de una empresa comercializadora de hortalizas en la región arroja buenos resultados, tanto desde el punto de vista financiero como de mercado. No obstante, para la implementación del proyecto es importante tener en cuenta no solo los aspectos financieros del mismo, sino el impacto que 
Tabla 6. Flujo de fondos (en pesos colombianos) proyectado para el proyecto de creación de Coophortiboy, 2011.

\begin{tabular}{|c|c|c|c|c|c|c|}
\hline & Año 0 & Año 1 & Año 2 & Año 3 & Año 4 & Año 5 \\
\hline \multicolumn{7}{|l|}{ Ingresos } \\
\hline Comisión venta & & 67.872 .000 & 79.094 .400 & 87.003 .840 & 95.704 .010 & 95.704 .010 \\
\hline Crédito Finagro & & 16.120 .000 & & & & \\
\hline Recursos propios & & 3.292 .098 & & & & \\
\hline Total ingresos & & 87.284 .098 & 79.094 .400 & 87.003 .840 & 95.704 .010 & 95.704 .010 \\
\hline \multicolumn{7}{|l|}{ Egresos } \\
\hline \multicolumn{7}{|l|}{ Inversiones } \\
\hline Maquinaria y equipo & 7.651 .500 & & & & & \\
\hline Capital de trabajo & 8.760 .598 & & & & & \\
\hline Costo de instalación & 3.000 .000 & & & & & \\
\hline Total inversiones & 19.412 .098 & & & & & \\
\hline \multicolumn{7}{|l|}{ Costos } \\
\hline \multicolumn{7}{|l|}{ Costo fijo } \\
\hline Mano de obra & & 39.744 .000 & 39.744 .000 & 39.744 .000 & 39.744 .000 & 39.744 .000 \\
\hline Servicios públicos & & 3.000 .000 & 3.000 .000 & 3.000 .000 & 3.000 .000 & 3.000 .000 \\
\hline Arrendamiento bodega & & 12.000 .000 & 12.000 .000 & 12.000 .000 & 12.000 .000 & 12.000 .000 \\
\hline Gastos de viaje & & 1.440 .000 & 1.440 .000 & 1.440 .000 & 1.440 .000 & 1.440 .000 \\
\hline Amortización & & 0 & 4.030 .000 & 4.030 .000 & 4.030 .000 & 4.030 .000 \\
\hline Interés crédito & & 2.129 .452 & 2.129 .452 & 1.597 .089 & 1.064 .726 & 532.363 \\
\hline Papelería & & 1.200 .000 & 1.200 .000 & 1.200 .000 & 1.200 .000 & 1.200 .000 \\
\hline Total costo fijo & & 59.513 .632 & 63.543 .452 & 63.011 .089 & 62.478 .726 & 61.946 .363 \\
\hline \multicolumn{7}{|l|}{ Costo variable } \\
\hline Transporte hortalizas & & 16.128 .000 & 16.128 .000 & 16.128 .000 & 16.128 .000 & 16.128 .000 \\
\hline Acondicionamiento hortalizas & & 2.688 .000 & 2.688 .000 & 2.688 .000 & 2.688 .000 & 2.688 .000 \\
\hline Mantenimiento equipos & & 1.200 .000 & 1.200 .000 & 1.200 .000 & 1.200 .000 & 1.200 .000 \\
\hline Total costo variable & & 19.809 .196 & 19.809 .196 & 19.809 .196 & 19.809.196 & 19.809 .196 \\
\hline Total costos & & 79.322 .828 & 83.352 .648 & 82.820 .285 & 82.287 .922 & 81.755 .559 \\
\hline Total egresos & -19.412 .098 & 79.322 .828 & 83.352 .648 & 82.820 .285 & 82287922 & 81.755 .559 \\
\hline Beneficios netos & -19.412 .098 & 7.961 .270 & -4.258 .248 & 4.183 .555 & 13.416.478 & 13.948 .451 \\
\hline Depreciaciones & & 697.500 & 697.500 & 697.500 & 697.500 & 697.500 \\
\hline Flujo neto de fondos & -19.412 .098 & 8.034 .020 & -3.561 .628 & 4.880 .075 & 14.112 .978 & 14.644 .951 \\
\hline
\end{tabular}

Tabla 7. Indicadores de rentabilidad y análisis de sensibilidad del estudio de factibilidad de Coophortiboy.

\begin{tabular}{|l|c|c|c|}
\hline \multicolumn{1}{|c|}{ Indicador } & Escenario inicial & Escenario disminución tarifa 10\% & Escenario incremento 10\% tarifa \\
\hline VAN & 9.520 .835 & -7.450 .688 & 10.841 .118 \\
\hline TIR (\%) & 21 & -- & 32 \\
\hline R B/C & 1,02269053 & 0,804998 & 1,069398 \\
\hline
\end{tabular}


a nivel socioeconómico genera en la población beneficiaria, tanto de productores como de consumidores. Así, este trabajo cumple con lo afirmado por Mokate (2009) que el estudio socioeconómico recoge información relevante acerca de los diferentes aspectos relacionados con las condiciones sociales de los grupos afectados por el proyecto y los impactos en el bienestar que pueda causar él mismo.

En los productores permitirá mejorar tanto sus condiciones económicas, como técnicas con la organización y planeación de la producción; de igual manera los consumidores se beneficiarán con un producto de mejor calidad y más sano.

De otra parte la organización de una entidad solidaria para los pequeños productores del DRACH, se convertirá también en un proyecto piloto en la región debido a que se pretende vincular a los productores con menores posibilidades de mercadeo a la comercializadora generando unas mejores condiciones de vida.

Otro aspecto que se debe tener en cuenta en el proyecto es el impacto ambiental que puede causar esta actividad en cada una de las etapas del proceso de adecuación y comercialización, teniendo en cuenta que el estudio ambiental mide el impacto que el proyecto puede tener sobre el medio ambiente y el entorno, lo mismo que las medidas para mitigar el daño que pueda producir en su ejecución (Miranda, 2011).

Como la función misional de la empresa se centra en la recepción de hortalizas previamente seleccionadas en las fincas, estas llegan a la planta libres de tierra adherida a las mismas, y sin la mayoría de las hojas que recubren la inflorescencia (en el caso del brócoli).

Por consiguiente, teniendo en cuenta que las actividades que se van a realizar dejan un mínimo de residuos sólidos de fácil evacuación, se puede afirmar que el daño que se ocasiona con este proyecto es mínimo.

\section{CONCLUSIONES}

La creación de una cooperativa integral de comercialización se puede convertir en una alternativa eficaz en la solución del problema de mercadeo de los productos, hortícolas (cebolla y brócoli) del DRACH, siempre y cuando se adelante el modelo asociativo en el marco de la cadena agrolimentaria.

La empresa que se conformará será de servicios y en consecuencia realizará una función de agente comercializador entre el asociado y el consumidor final.

Las hortalizas como el brócoli y la cebolla, se constituyen en una alternativa competitiva y sostenible si la producción se desarrollan con calidad, volumen, continuidad y criterio empresarial.

Bajo una organización de carácter solidaria como Coophortiboy se puede regular precios, eliminar intermediarios y reducir costos de producción, convirtiéndose en una alternativa para desarrollar operaciones de poscosecha como adecuación yempaque.

En este modelo de organización se permitirá la amplia participación de los productores, quienes se beneficiarán no solo en la comercialización sino también en la producción, al implementar programas productivos de acuerdo con las necesidades del consumidor.

Coophortiboy, por ser una cooperativa integral, podrá implementar hacia el futuro otra serie de actividades relacionadas con su objeto social, involucradas con la cadena productiva hortícola apoyada en iniciativas de solidaridad y educación propias del modelo cooperativo. 


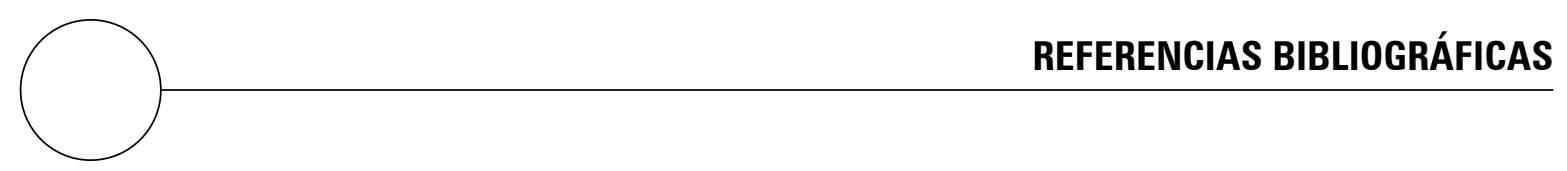

Agronet. 2013. Brócoli. En: http://www.agronet.gov.co; consulta: 15 de abril de 2013.

Córdoba P., M. 2006. Formulación y evaluación de proyectos. ECOE Ediciones, Bogotá.

CCI. 2000. Estudio de mercado de hortalizas. Corporación Colombia Internacional, Bogotá.

DNP. 2012. Manual de soporte conceptual para el uso de la metodología general para la formulación y evaluación de proyectos. Dirección de Inversiones y Finanzas Públicas. Departamento Nacional de Planeación, Bogotá.

ILPES. 1973. Guía para la presentación de proyectos. Instituto Latinoamericano de Planificación Económica y Social, México.

Miranda, J.J. 2011. Gestión de proyectos. Alfaomega, Bogotá.

Mokate, K.M. 2009. Evaluación financiera de proyectos de inversión. Alfaomega, Bogotá.

Murcia, H. 1995. Administración de empresas asociativas de producción agropecuaria. IICA, San José.
Murcia, J.D., F.N. Díaz, G. Oñate, C. Ortega, L. Santana, M.R. González, V. Medelín y C. Baca. 2009. Proyectos: formulación y criterios de evaluación. Alfaomega, Bogotá.

Naranjo P., M. 2008. Proyectos sociales. Uniminuto, Bogotá.

Nuñez Z., R. 2007. Manual para la evaluación de proyectos de inversión. Trillas, México.

Pineda S., C.J. 2008. Cómo organizar empresas solidarias eficientes. Editorial Antropus, Bogotá.

Prieto H., J.E. 2009. Proyectos enfoque gerencial. ECOE Ediciones, Bogotá.

Ramírez, J.A. 2011. Evaluación financiera de proyectos. Ediciones de la U, Bogotá.

Rojas L., M.D. 2007. Evaluación de proyectos para ingenieros. ECOE Ediciones Bogotá.

Usochicamocha. 2011. Informe de gestión. Duitama, Colombia. 